\title{
Suppression of Spin Projection Noise in Broadband Atomic Magnetometry
}

\author{
JM Geremia, ${ }^{*}$ John K. Stockton, and Hideo Mabuchi \\ Physics and Control \& Dynamical Systems, California Institute of Technology, Pasadena California 91125, USA
}

(Received 2 September 2003; revised manuscript received 15 February 2005; published 24 May 2005)

\begin{abstract}
We demonstrate that quantum nondemolition measurement, combined with a suitable parameter estimation procedure, can improve the sensitivity of a broadband atomic magnetometer by reducing uncertainty due to spin projection noise. Furthermore, we provide evidence that real-time quantum feedback control offers robustness to classical uncertainties, including shot-to-shot atom number fluctuations, that would otherwise prevent quantum-limited performance.
\end{abstract}

DOI: 10.1103/PhysRevLett.94.203002

PACS numbers: 32.80.Qk, 06.20.-f, 32.80.Pj

An atomic magnetometer estimates the magnitude of a magnetic field by observing Larmor precession in a spinpolarized atomic sample [1]. Therefore, a canonical procedure for "detecting" a magnetic field along the laboratory $y$ axis operates by preparing the collective magnetic moment of $N$ atoms to lie along the $x$ axis. The experimenter then watches the $z$ component of that magnetic moment for signatures of $y$ axis Larmor precession.

More specifically, the spin-polarized atomic system is characterized by its net magnetization $\mathbf{F} \equiv\langle\hat{\mathbf{F}}\rangle$, where $\hat{\mathbf{F}}$ is the quantum operator for the collective atomic angular momentum. Under the influence of the magnetic field $\mathbf{b}=$ $B \hat{\mathbf{y}}, \mathbf{F}$ precesses from the $x$ axis,

$$
d \mathbf{F}(t)=-\gamma(\mathbf{F}(t) \times \mathbf{b}) d t, \quad \mathbf{F}(0)=\hbar F \hat{\mathbf{x}},
$$

where $F=N f$ for $N$ spin- $f$ atoms and the Larmor frequency $\omega_{\mathrm{L}}=\gamma B$ is determined by the gyromagnetic ratio $\gamma$. These dynamics confine the mean spin vector to the $x z$ plane such that, in the small-time (and small-decoherence) limit appropriate for discussing detection thresholds, the $z$ component of the atomic magnetization is given by $F_{\mathrm{z}}(t)=\gamma B F t, t \ll \omega_{L}^{-1}$.

The magnetic field can thus be inferred from the slope of $F_{\mathrm{z}}$ during its small-angle Larmor precession,

$$
\tilde{B}=\frac{1}{\gamma F}\left(\frac{F_{\mathrm{z}}(t)}{t}\right) \rightarrow \frac{1}{\gamma F} F_{\mathrm{z}}^{\prime}, \quad 0 \leq t<\tau .
$$

Uncertainty in the field estimation $\Delta \tilde{B}$ results from various sources of error that can be divided into three classes: (1) spin projection noise [2], or quantum uncertainty in the initial orientation of $\mathbf{F}$ due to noncommutativity of the quantum operators $\hat{F}_{\mathrm{x}}, \hat{F}_{\mathrm{y}}$, and $\hat{F}_{\mathrm{z}}$, (2) finite signal to noise in the physical measurement used to determine $F_{\mathrm{z}}$, and (3) classical parameter uncertainties in Eq. (2), namely, fluctuations in $F$ that arise from shot-to-shot variance in the atom number $N$.

Here we demonstrate that, given a quantum nondemolition (QND) measurement of $F_{\mathrm{z}}$ with a finite signal to noise ratio, degradation of the field sensitivity due to projection noise can be minimized by an estimation procedure [3,4] that exploits the spin squeezing produced by the QND measurement [5-7]. However, we find that the simplest procedure for suppressing spin projection noise is susceptible to classical parameter uncertainty. Incorporating realtime quantum feedback control into the estimation procedure alleviates this source of error.

We consider a QND measurement of $F_{\mathrm{z}}$ performed by quantum-limited detection of an optical field scattered by the atomic system $[7,8]$. Such a measurement is described by the continuous photocurrent

$$
y(t)=\sqrt{S} F_{\mathrm{z}}(t)+\zeta(t),
$$

where the $\zeta(t)$ are Gaussian stochastic increments that reflect detection (optical) noise. The coefficient $S$ relates the mean value of the photocurrent to the $z$ component of the collective atomic spin [7].

Our procedure [3], based on quantum Kalman filtering $[9,10]$, estimates the magnetic field from the average slope $\bar{y}^{\prime}(\tau)$ obtained by regressing the QND photocurrent over the interval $0 \leq t \leq \tau$. Figure 1 schematically illustrates this procedure. Beginning from the spin-polarized state at $t=0$, the measurement reveals both the slope due to (small-angle) Larmor precession and an offset due to the initial uncertainty in the orientation of $\mathbf{F}$. This spin projection noise offset is randomly distributed with variance $\Delta \hat{F}_{\mathrm{z}}^{2}(0) \approx F / 2$ in an ensemble of measurement trajectories, according to the Heisenberg-Robertson relation $\Delta \hat{F}_{\mathrm{y}}^{2} \Delta \hat{F}_{\mathrm{z}}^{2} \geq \frac{1}{4} \hbar^{2}\left|\left\langle\hat{F}_{\mathrm{x}}\right\rangle\right|$. Freedom to shunt the random offset

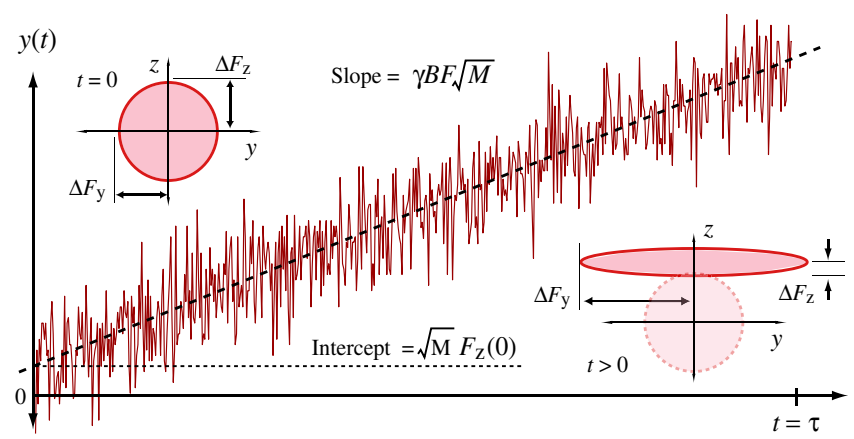

FIG. 1 (color online). Atomic magnetometry based on continuous QND measurement and quantum filtering enables field estimation procedures that suppress projection noise of the initial atomic state (illustrative simulation). 
$F_{\mathrm{z}}(0)$ into the regression intercept rather than the slope, gained simply by performing a two-parameter fit, minimizes the impact of quantum uncertainty on the field estimate.

Our single-shot magnetic field estimate is given by

$$
\tilde{B}(\tau)=\frac{\bar{y}_{\tau}^{\prime}}{\gamma F \sqrt{S}}, \quad \tau \ll \omega_{L}^{-1},
$$

where $\bar{y}_{\tau}^{\prime}$ is the photocurrent slope obtained by linear regression of $y(t)$ over the time interval $0 \leq t<\tau$. In principle, field uncertainty is limited only by statistical regression error [3]

$$
\Delta \tilde{B}(\tau)=\frac{1}{\gamma F \tau} \sqrt{\frac{3 \Delta \zeta_{\tau}^{2}}{S}}
$$

where $\Delta \zeta_{\tau}^{2} \equiv \mathbb{E}\left[\frac{1}{\tau} \int_{0}^{\tau} \zeta(t) d t\right]^{2}$ is the integrated noise variance in a $1 / \tau$ bandwidth. The unitless QND signal to noise ratio SNR $=\sqrt{S} / \Delta \zeta_{\tau}$ (both $S$ and $\Delta \zeta_{\tau}^{2}$ have units proportional to frequency), is determined by experimental parameters, such as the optical probe power and detuning, and the scattering interaction strength [7].

The uncertainty of our optimal estimator, Eq. (5), should be compared to that of a procedure that cannot distinguish between Larmor precession and the initial spin projection noise. Such is the case for steady-state atomic magnetometers $[11,12]$ where the uncertainty

$$
\Delta \tilde{B}(\tau)=\frac{2}{\gamma F \tau} \sqrt{\Delta F_{\mathrm{z}}^{2}(0)+\frac{\Delta \zeta_{\tau}^{2}}{S}}
$$

retains a contribution from both $\Delta \hat{F}_{\mathrm{Z}}^{2}(0) \approx F / 2$ and the optical shotnoise. In the limit of infinite signal to noise this expression saturates to the so-called shotnoise magnetometry limit [11]. Equation (6) corresponds to an estimation procedure that averages the photocurrent

$$
\tilde{B}(\tau)=\frac{2}{\gamma F \tau^{2} \sqrt{S}} \int_{0}^{\tau} y(t) d t
$$

rather than determining its slope. It is readily shown that steady-state atomic magnetometers operate in a manner logically equivalent to this type of direct averaging.

Our estimation procedure, which suppresses projection noise, requires precise knowledge of the QND measurement sensitivity $F \sqrt{S}$. Shot-to-shot variation in $N$ produces fluctuations, $\Delta F$, in the length of $\mathbf{F}$ that directly propagate into the field estimation as a proportional error $\Delta \tilde{B}_{\mathrm{F}}=$ $\bar{y}^{\prime} \Delta F /(\gamma F \sqrt{S}) \approx B(\Delta F / F)$. A similar argument applies to $S$. While relative parameter uncertainties introduce essentially no error when $B=0$, they can completely mask the improved resolution provided by spin squeezing when $B \neq 0$.

To reduce the effects of classical parameter uncertainty, our magnetometer is implemented according to the closedloop methodology [4] illustrated in Fig. 2. The QND photo- current $y(t)$ drives a precision $y$-axis magnet in negative feedback configuration to stabilize $F_{\mathrm{z}}$ to zero $[8,13]$. In the presence of an external magnetic field, the controller imposes a compensating field $\mathbf{b}_{\mathrm{c}}(t) \simeq-B(t) \hat{\mathbf{y}}$ to prevent the atomic magnetization from precessing out of the $x y$ plane. The magnetic field is estimated from the time-averaged feedback signal

$$
\tilde{B}(\tau)=-\frac{1}{\tau} \int_{0}^{\tau} B_{\mathrm{c}}(t) d t
$$

rather than the photocurrent. Since the magnetometer always operates with $F_{\mathrm{z}} \sim 0$, the closed-loop estimation is reasonably immune to atom number fluctuations.

We have recently demonstrated QND detection and realtime quantum-limited feedback control with an apparatus similar to that in Fig. $2[7,13]$. Our spin system is provided by the $6^{2} S_{1 / 2}(F=4)$ ground state hyperfine manifold in ${ }^{133}$ Cs. We obtain samples with $N \sim 10^{10}-10^{11}$ atoms at a temperature of $T=10 \mu \mathrm{K}$ via dark spontaneous-force optical trapping. Shot-to-shot fluctuations in $N$ are $<20 \%$. Spin polarization along the $x$ axis is achieved by optical pumping on the $6^{2} S_{1 / 2}(F=4) \rightarrow 6^{2} \mathrm{P}_{3 / 2}\left(F^{\prime}=4\right)$ hyperfine transition and continuous QND measurement of $F_{\mathrm{Z}}$ is implemented by balanced polarimetric detection of a laser detuned from the $4 \rightarrow 5$ transition by $\Delta=550 \mathrm{MHz}$.

Background magnetic fields are continually nulled using a combination of large $(1 \mathrm{~m})$ external three-axis Helmholtz coils and smaller computer controlled trim coils. The experiment is synchronized with respect to the $60 \mathrm{~Hz}$ line

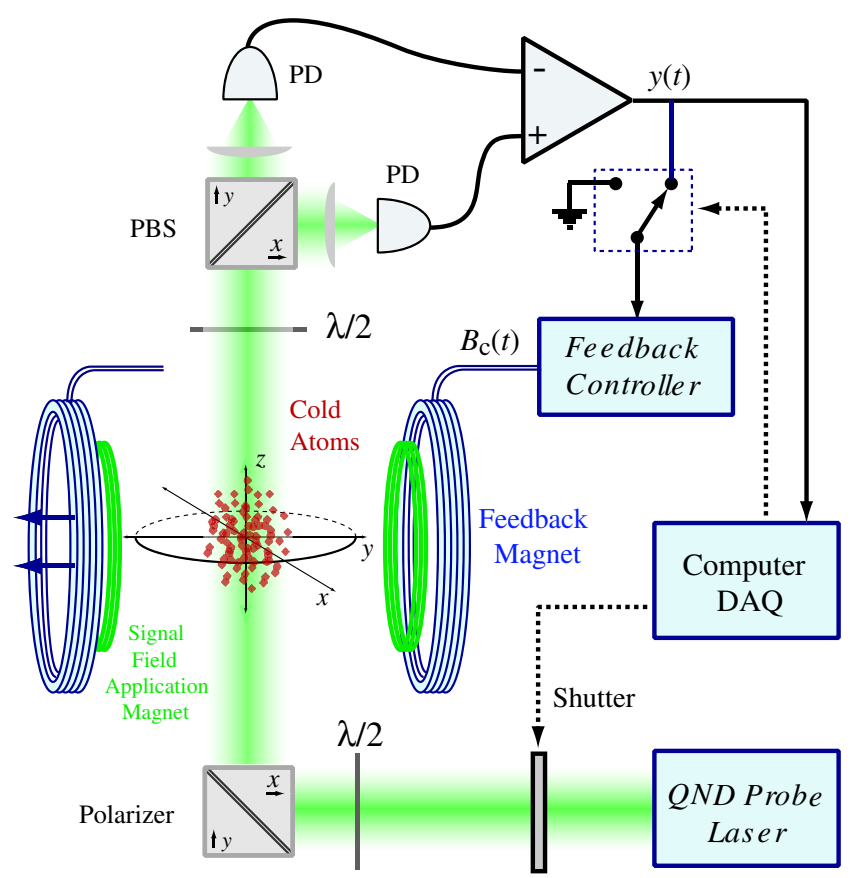

FIG. 2 (color online). Schematic of our apparatus for broadband atomic magnetometry based on continuous QND measurement and real-time quantum feedback control. 

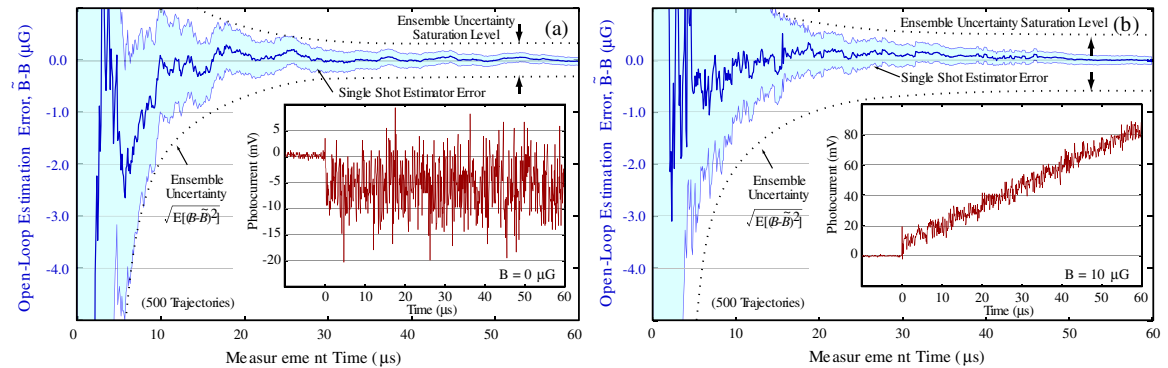

FIG. 3 (color online). Open-loop magnetic field estimation error, $\tilde{B}-B$, as a function of measurement time for (a) $B=0$ and (b) $B=10 \mu \mathrm{G}$. Inset plots show the polarimeter photocurrent for each QND measurement trajectory. The shaded regions indicate the single-shot regression uncertainty while the dotted curves reflect the ensemble error measured from 500 trajectories.

cycle, and we estimate the resulting shot-to-shot field fluctuations in a $100 \mu \mathrm{s}$ measurement window to be $\sim 850 \mathrm{nG}$. Atomic decoherence is $<6 \%$ over the $t=$ $100 \mu \mathrm{s}$ measurement trajectories we consider [7]. Further characterization of our state preparation, atom number, transverse spin relaxation, spin squeezing, and quantum noise limited feedback performance can be found in Ref. [13]. A detailed procedure for determining the degree of atomic polarization and the QND signal to noise ratio can be found in Ref. [7].

We began by operating our magnetometer with feedback disabled in order to characterize the adverse effects of classical parameter uncertainty. Figure 3 shows example open-loop field estimations performed using the procedure in Eq. (4) for two different magnetic fields, $B=0$ and $B=$ $10 \mu \mathrm{G}$. When the QND measurement is initiated at $t=0$ by opening the probe laser shutter [refer to Fig. 2] the photocurrent establishes an average offset [inset of Fig. 3(a)] that is randomly distributed in an ensemble of similar trajectories. Our ability to observe this random offset reflects sufficient signal to noise in our QND measurement to produce squeezing $[3,7,13]$.

Since $B=0$ in Fig. 3(a), the atoms do not undergo Larmor precession and the slope of $y(t)$ is, as expected, $\bar{y}^{\prime} \sim 0$. As described above, statistical fluctuations due to optical noise require that this slope be obtained by regression, as filtering the photocurrent reduces the stationary noise by time averaging. The single-shot estimation trajectory for $B=0$ computed according to Eq. (4) is depicted by the dark solid line in Fig. 3(a) while the light shaded region denotes the single-shot field uncertainty, $\Delta \tilde{B}$, due to statistical error in the linear regression. Values for $F$ and $S$ needed to evaluate Eq. (4) were obtained from full-scale atomic Larmor precession according to the procedure detailed in Ref. [7].

The dotted lines in Fig. 3(a) indicate the ensemble field variance, computed as $E\left[(\tilde{B}-B)^{2}\right]^{1 / 2}$ from 500 QND trajectories, for the $B=0$ field estimate. At long times, this measure of the magnetometer performance saturates to the level of shot-to-shot background magnetic field fluctuations in our experimental apparatus, approximately $850 \mathrm{nG}$. However, prior to saturation, as depicted by the
$B=0$ curves in Fig. 4, the regression estimation procedure (circles) outperforms the direct averaging estimator (triangles) given by Eq. (7). Unlike direct averaging, the regression estimator suppresses the uncertainty due to initial spin projection noise - the ensemble uncertainty drops below the field uncertainty threshold given by Eq. (6) [dotted line in Fig. 4].

It is important to note that the coherent state projection uncertainty (dotted line in Fig. 4) was computed using an absolute calibration [7] of $S$, and the average value of $F$ inferred from full-amplitude Larmor precession measurements. Even though our optically pumped atomic system did not likely begin from a true minimum-uncertainty state due to imperfect pumping, sufficient QND spin noise reduction was achieved to allow the magnetometer to outperform the projection noise uncertainty corresponding to that of an actual coherent state.

In contrast, the $B=10 \mu \mathrm{G}$ open-loop estimation uncertainty fails to surpass the coherent state threshold despite a

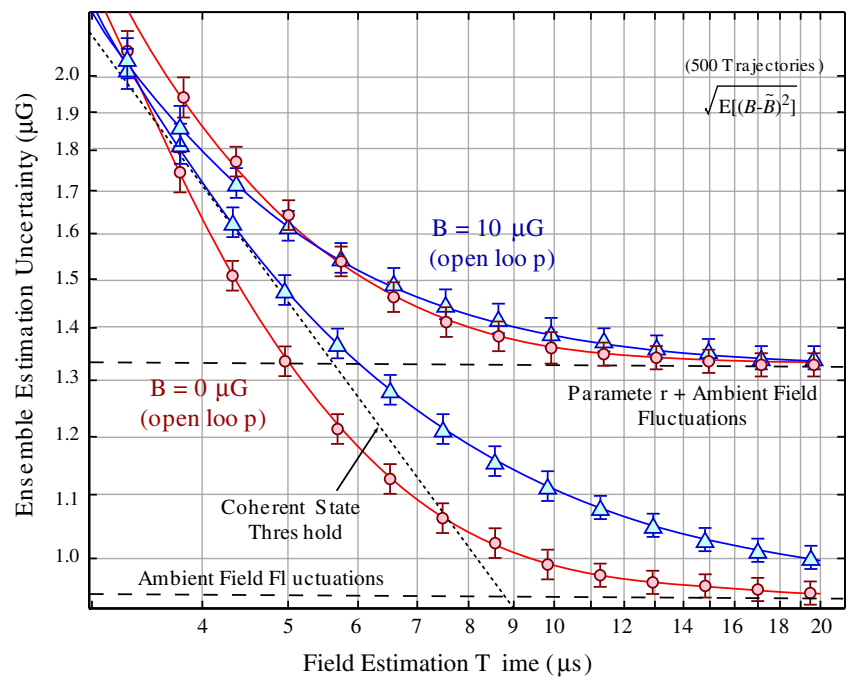

FIG. 4 (color online). Comparison of the two estimation procedures, Eqs. (4) (circles) and (7) (triangles), with $B=0$ and $B=10 \mu \mathrm{G}$. The dotted line reflects the theoretical sensitivity limit, Eq. (6), of a magnetometer with the same signal to noise ratio but that does not exploit spin squeezing. 

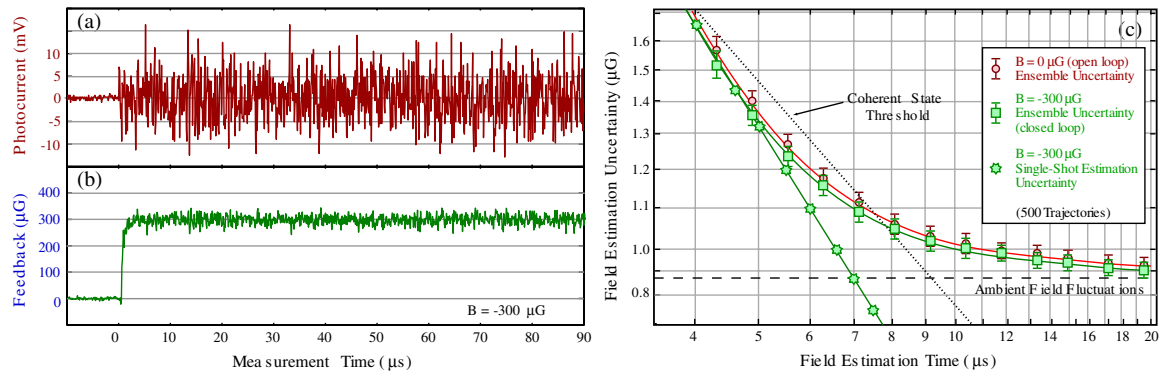

FIG. 5 (color online). (a) Closed-loop photocurrent for a $B=-300 \mu \mathrm{G}$ field, (b) real-time feedback field, and (c) the resulting closed-loop field estimation error $\tilde{B}-B$ as a function of measurement time.

clearly visible photocurrent offset [inset in Fig. 3(b)] suggesting the presence of spin squeezing. Evidently, the nonzero slope renders the open-loop estimation susceptible to classical parameter uncertainty in $F$ and $S$. As such, the long time estimation uncertainty for $B=10 \mu \mathrm{G}$ saturates to a level much higher than that of the ambient magnetic field fluctuations, as in Fig. 4.

To alleviate the effects of classical parameter uncertainty, we next performed our closed-loop estimation procedure by enabling the feedback loop for the entire duration of each QND trajectory. The photocurrent in Fig. 5(a) displays no discernable slope despite the presence of a $B=-300 \mu \mathrm{G}$ field as the feedback loop drives a cancellation field [Fig. 5(b)] $B_{\mathrm{c}}$ to maintain $F_{\mathrm{z}} \sim 0$. The closed-loop field estimate, computed according to Eq. (8) for $0 \leq t \leq \tau$, is seen to be robust to shot-to-shot parameter fluctuations - it is evident from Fig. 5(c) that the ensemble uncertainty of the closed-loop estimator for $B=-300 \mu \mathrm{G}$ (squares) achieves similar performance to the $B=0$ open-loop estimation (circles). Despite the large magnitude of the estimated field, the closed-loop procedure is able to outperform the coherent state projection noise threshold [dotted line in Fig. 5(c)].

It should also be pointed out that in the closed-loop configuration, where the estimation uncertainty is due almost entirely to QND detection noise, the ensemble variance is an overly conservative measure of the magnetometer performance. After all, ambient fluctuations that produce the $850 \mathrm{nG}$ sensitivity floor in Figs. 4 and 5(c) are real magnetic fields sensed by the atoms. Where other contributions to the detection threshold are well controlled, the single-shot estimation error [stars in Fig. 5(c) ] more accurately reflects the magnetometer's performance. This single-shot closed-loop uncertainty surpasses the coherent spin state threshold at even long times in this case prior to the onset of significant atomic decoherence.

These results highlight what we anticipate will become a central theme in quantum-limited metrology. Feedback enables a precision measurement to achieve optimal insensitivity to classical uncertainty without sacrificing resolution $[4,14]$. Furthermore, our closed-loop methodology can be immediately extended to detection of nonstationary fields. Such an approach is likely to be essential for obtaining acceptable performance in various precision metrological applications including spin resonance measurements, atomic frequency standards, and matter-wave gravimetry.

This work was supported by the NSF (PHY-9987541, EIA-0086038), the ONR (N00014-00-1-0479), and the Caltech MURI Center for Quantum Networks (DAAD1900-1-0374). J. K. S. acknowledges the Hertz Foundation. We thank Ramon van Handel, Andrew Berglund, Michael Armen, Andrew Doherty, Dima Budker, and Dan Kleppner and Vladan Vuletic for helpful discussions.

*Electronic address: jgeremia@Caltech.EDU

[1] J. Dupont-Roc, S. Haroche, and C. Cohen-Tannoudji, Phys. Lett. A28, 638 (1969).

[2] W. Itano, J. Berquist, J. Bollinger, J. Gilligan, D. Heinzen, F. Moore, M. Raizen, and D. J. Wineland, Phys. Rev. A 47, 3554 (1993).

[3] JM Geremia, J.K. Stockton, A.C. Doherty, and H. Mabuchi, Phys. Rev. Lett. 91, 250801 (2003).

[4] J. K. Stockton, J.M. Geremia, A. Doherty, and H. Mabuchi, Phys. Rev. A 69, 32109 (2004).

[5] A. Kuzmich, L. Mandel, and N. P. Bigelow, Phys. Rev. Lett. 85, 1594 (2000).

[6] B. Julsgaard, A. Kozhekin, and E.S. Polzik, Nature (London) 413, 400 (2001).

[7] JM Geremia, J. K. Stockton, and H. Mabuchi, quant-ph/ 0501033 [Phys. Rev. A (to be published)]. (Note that the data presented in Ref. [7] was acquired after an overhaul of the apparatus utilized here and in Ref. [13]).

[8] L. K. Thomsen, S. Mancini, and H. M. Wiseman, Phys. Rev. A 65, 061801 (2002).

[9] V.P. Belavkin, Rep. Math. Phys. 43, A405 (1999).

[10] H. Mabuchi, Quantum Semiclass. Opt. 8, 1103 (1996).

[11] D. Budker, W. Gawlik, D. Kimball, S. Rochester, V. Yashchuk, and A. Weiss, Rev. Mod. Phys. 74, 1153 (2002).

[12] I. K. Kominis, T. W. Kornack, J.C. Allred, and M. Romalis, Nature (London) 422, 596 (2003).

[13] JM Geremia, J. K. Stockton, and H. Mabuchi, Science 304, 270 (2004).

[14] D. W. Berry and H. M. Wiseman, Phys. Rev. A 65, 043803 (2002). 